\title{
Adaptive pixel mass recalibration for mass spectrometry imaging based on locally endogenous biological signals.
}

Raphaël La Rocca*, „, , Christopher Kune ${ }^{\dagger}$, Mathieu Tiquet ${ }^{\dagger}$, Lachlan Stuart, Gauthier Eppe $^{\dagger}$, Theodore Alexandrov ${ }^{\ddagger}$, Edwin De Pauw ${ }^{\dagger}$ and Loïc Quinton ${ }^{\dagger}$

Mass Spectrometry Laboratory, MolSys Research Unit, University of Liège, Liège, Belgium

†Structural and Computational Biology Unit, European Molecular Biology Laboratory, Heidelberg, Germany.

§Skaggs School of Pharmacy and Pharmaceutical Sciences, University of California at San Diego, San Diego, California 92093, United States

\begin{abstract}
Mass spectrometry imaging (MSI) is a powerful and convenient method for revealing the spatial chemical composition of different biological samples. Molecular annotation of the detected signals is only possible if a high mass accuracy is maintained over the entire image and the $\mathrm{m} / \mathrm{z}$ range. However, the heterogeneous molecular composition of biological samples could lead to small fluctuations in the detected $\mathrm{m} / \mathrm{z}$-values, called mass shift. The use of internal calibration is known to offer the best solution to avoid, or at least to reduce, mass shifts. Their "a priori" selection for a global MSI acquisition is prone to false positive detection and therefore to poor recalibration. To fill this gap, this work describes an algorithm that recalibrates each spectrum individually by estimating its mass shift with the help of a list of pixel specific internal calibrating ions, automatically generated in a dataadaptive manner (https://github.com/LaRoccaRaphael/MSI_recalibration). Through a practical example, we applied the methodology to a zebrafish whole body section acquired at high mass resolution to demonstrate the impact of mass shift on data analysis and the capability of our algorithm to recalibrate MSI data. In addition, we illustrate the broad applicability of the method by recalibrating 31 different public MSI datasets from METASPACE from various samples and types of MSI and show that our recalibration significantly increases the numbers of METASPACE annotations (gaining from 20 up to 400 additional annotations), particularly the high-confidence annotations with a low false discovery rate.
\end{abstract}




\section{Introduction}

In biology and medicine, the in-situ determination of the molecular environment is of prime importance to understand biological processes and pathology evolution ${ }^{1}$. This growing interest pushes the development of analytical methods that correlate spatial distribution with the detection of different biological molecules such as peptides ${ }^{2}$, metabolites and lipids ${ }^{3}$. Mass spectrometry imaging (MSI) has been demonstrated particularly powerful as it can rapidly reveal, in an untargeted manner, a wide range of compounds present in small amounts in biological samples as various as whole-body sections $^{4,5}$, tissue sections, bacteria colonies ${ }^{6}$, plants ${ }^{7}$ or again single cells ${ }^{8,9}$. MSI is a particular application of mass spectrometry (MS) in which spectra are recorded, usually by MALDI or, to a lesser extent DESI ${ }^{10}$ and SIMS $^{11}$, at different positions, called pixels, over a sample forming a 2D image. A pixel is then a spectrum identified by its coordinates $(x, y)$, containing $m / z$ values (channels) and their intensities ${ }^{12,13}$.

While high mass resolution is required to distinguish isobaric and quasi-isobaric compounds in complex mixtures and to exploit isotope signatures, high accuracy of $\mathrm{m} / \mathrm{z}$ measurement is essential for reliable compound identification. For this reason, high resolution mass spectrometry (HRMS), combining both high resolution and mass accuracy, is encouraged for the annotation of molecular signatures of biological samples ${ }^{14}$. However, the MSI data acquired by HRMS have shown that they suffer from an inconsistent variation of measured $\mathrm{m} / \mathrm{z}$ values, from pixel to pixel. There are various reasons for mass shift phenomenon in MSI, most of which depend on the design of the mass analyzer. For example, it has been shown that the number of ions in Fouriertransform ion cyclotron resonance cell (FT-ICR) is correlated with mass shifts ${ }^{15}$. The analysis of MSI images is based on a so-called average spectrum, representing all the ions detected in the image, i.e. by summing each spectrum in each pixel. The pixel-to-pixel fluctuation of measured $\mathrm{m} / \mathrm{z}$ reaches up to several ppm and strongly influences the accuracy and resolution of the MSI average spectra $^{16-18}$. It thus has a crucial impact on MSI interpretation. Indeed, mass shifts affect the quality of the results by (i) decreasing the number of identifications, (ii) increasing the number of false identifications, (iii) reducing the confidence of each identification and (iv) having an impact on the ability to reconstruct the appropriate spatial distributions of specific detected species. It has already shown that automated annotation of metabolite for MSI data is critically dependent on $m / z$ accuracy and requires the accuracy of at least $3 \mathrm{ppm}$ in $m / z^{19}$. In summary, mass shifts strongly weaken the advantages of HRMS instruments for MSI data analysis.

The mass accuracy depends mainly on the quality of the MS calibration of the instrument, while the mass resolving power is linked to the mass analyzer device ${ }^{20}$. Instrument calibration is performed by locking the experimental $\mathrm{m} / \mathrm{z}$ signals to their theoretical $\mathrm{m} / \mathrm{z}$ values using a suitable mathematical function (e.g., linear, quadratic, or cubic functions). In general, the MS calibration function is estimated from the signal of a calibrating substance acquired separately from the MSI sample (i.e. external calibration) ${ }^{21}$. This function is then applied uniformly to each pixel. Since mass shift is a pixel-dependent effect, the application of a unique MS calibration function cannot reduce this phenomenon ${ }^{16,22}$. Recalibration of MSI data using individual spectrum recalibration functions estimated directly from specific signals in the spectrum (i.e. internal calibration) appears as a method of choice for removing the mass shift effect ${ }^{16,23}$. The signal of additive molecules ${ }^{16,18}$ or endogenous molecules (e.g. matrix signal) ${ }^{23,24}$ can be exploited to performed the MSI recalibration. The use of endogenous molecules has as the advantage of avoiding the ion suppression effect generated by the added reference molecules. In this approach, the set of potential calibrating ions has to be known in advance and has to be detected in each pixel to effectively and efficiently reduce the mass shift contribution ${ }^{17}$. This limits its application to highly homogenous MSI sample whose expected signal is already known (i.e. the signal of the matrix ions or of the ions already identified). Moreover, the application of this method to MSI involving complex spectra increases the chance to select inappropriate calibration ions.

Other approaches, such a MS spectra alignment method, have been investigated to counteract the mass shift phenomenon. However, in spite of the increase in spectra consistency, alignment does not necessarily correct for mass shifts, contrary to recalibration ${ }^{25-28}$.

In this context, this work aims at proposing a post-acquisition data-adaptive recalibration methodology to reduce mass shifts in MS data, based on a robust and an automated selection of adequate calibrating signals in each pixel, independently. In this case, the method is applied on MSI by using confidently identified lipids and metabolites from MSI datasets in METASPACE (open and free access platform) to constitute these calibrating ion lists.

\section{Experimental Section \\ Material}


In this work, two types of datasets have been analyzed: a zebrafish cryo-section and a set 31 public MSI datasets from METASPACE. First, a $12 \mu \mathrm{m}$ thick slices of one-month-old zebrafish embedded in gelatin were realized on the Cryostar NX70 (Thermo Scientific) then placed on an Indium Tin Oxide glass slide (ITO slide, Bruker, Bremen, Germany). All manipulations on zebrafish are approved by the Animal Welfare Committee of the University of Liège and are performed according to Belgian federal regulations for animal protection. After 15 minutes of desiccation, tissue slices were covered by CHCA matrix (97\% purity, Sigma-Aldrich, Taufkirchen, Germany) using an automatic sprayer SunCollect System (SunChrom). MSI acquisition was performed on a SolariX XR 9.4T (Bruker) using the automation software Flexlmaging 5.0 (Bruker, Bremen, Germany). Acquisition method consists of 400 laser shots per pixel fired at $1000 \mathrm{~Hz}$ with the laser power fixed at $70 \%$. The minimum laser focus was employed with a raster width of $60 \mu \mathrm{m}$ leading to images ranging from 10 to $15 \mathrm{k}$ pixels. The image was converted in centroided imzML ${ }^{29}$ format (common data format for $\mathrm{MSI}$ ) using Flexlmaging 5.0. On the other hand, 31 public MSI datasets were selected in METASPACE as representative for different ion sources (MALDI/DESI), analyzers (Orbitrap/FTICR), polarities (positive/negative) and MALDI matrices (DHB for positive mode, DAN for negative mode, CHCA/Norharmane for both modes). To represent a maximum of the METASPACE samples, we have selected human/mouse samples when possible. In addition, 6 datasets from Waters were included to cover several TOF-based analyzers. All the MSI were downloaded as centroided imzML ${ }^{29}$ (see S1 for more details). The metadata about the sample preparation was, however, only partially provided (see S1) and the parameters used to convert the raw image into centroided imzML are not known.

\section{Method}

\section{General approach}

In this work, the term "hit" is used to depict a match between experimental signal $m / z\left(m / z_{\text {exp }}\right.$.) and internal calibrating ion $\mathrm{m} / \mathrm{z}\left(\mathrm{m} / \mathrm{z}_{\text {calib }}\right)$ within a given mass tolerance from the $m / z_{\text {calib. We will call }}$ "mass error" the difference in mass between the $m / z_{\text {exp }}$ and its linked $m / z_{\text {calib }}$ (Equation 1 ). A hit is considered as true if the experimental signal corresponds to the calibration ion. Otherwise, an erroneous match between the experimental signal and the internal calibrating ion is a false hit. In MSI, the mass spectra are information-rich and often contain 10e4 peaks per spectrum.
Therefore, it's particularly difficult to discriminate true from false hits as multiple signals can be found in the mass range of a given $m / z_{\text {calib. This }}$ difficulty is amplified by the presence of mass shifts since higher mass tolerance is required. However, increasing the mass tolerance inevitably increases the number of false hits, decreasing the recalibration performances. Therefore, the algorithm presented in this work optimizes the selection of true hits for recalibrating each pixel individually. We assume that true hits have mass errors directly correlated to the mass shifts. Then, the calculated mass error is used as a criterion for the selection of hits, considering that hits with the most frequent mass errors are predominantly true. These hits are thus selected to fit a linear model of the mass errors according to $\mathrm{m} / \mathrm{z}$. Finally, the recalibration is performed by removing the estimated errors in every detected $m / z$ values.

This algorithm is divided into 5 steps (Figure 1 ). (1) The generation of the list of $\mathrm{m} / \mathrm{z}_{\text {calib. }}$ for the whole MSI data according to similar public MSI datasets from METASPACE (i.e. representing the same kind of biological samples) since we assume them to share metabolites with the sample of interest. (2) Centroid MS spectra are extracted from each pixel. (3) The calibrant hits are generated by computing the mass errors between the list of potential internal calibrating ions and the spectrum signals for each pixel. (4) The preferential calibrant hits are selected as those with the most frequent mass errors with the aim to select true hits. (5) A linear model for predicting the mass shifts based on the preferential hits is then constructed and applied to all spectra for their recalibration.

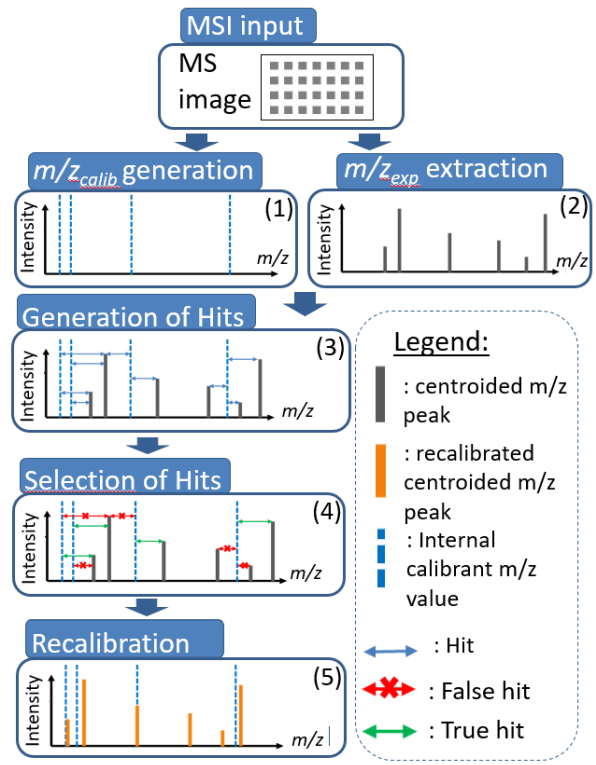

Figure 1. Overview of the recalibration algorithm. 


\section{Generation of the list of internal calibrating ions}

Generating the $m / z_{\text {calib. }}$ list covering the signals from the sample of interest is a critical step for accurate estimation of mass shifts as it directly influences the collection of true hits. Therefore, for each MSI data subject to recalibration, a list of annotations from similar public METASPACE datasets is generated. However, it is not mandatory to use METASPACE to generate the $\mathrm{m} / \mathrm{z}_{\text {calib }}$ as any list of $\mathrm{m} / \mathrm{z}$ values could work, yet using METASPACE will result in fewer false hits.

\section{Generating the Hits}

The $m / z_{\text {exp }}$ list is made of the detected peaks, for each pixel (Figure 1.2). The $m / z_{\text {exp }}$ list are then compared to the list of $\mathrm{m} / \mathrm{z}_{\text {calib. }}$, within a mass tolerance called T1 in (Figure 1.3). This mass tolerance window is considered large enough to encompass most of the extreme mass shifts in the data, and small enough to discard the contribution of non-relevant peaks and isotopes. The mass errors in $\mathrm{Da}$, used to calculate the regression and the error distribution, are calculated for each hit using Equation 1 where $M_{\text {error, }} M_{\text {exact }}$ and $M_{\text {experiment }}$ are, respectively, the mass error, the exact mass (from $\mathrm{m} / \mathrm{z}_{\text {calib. }}$ list) and the experimental mass (from the $\mathrm{m} / \mathrm{z}_{\text {exp }}$. list).

$$
\begin{gathered}
M_{\text {error }}=M_{\text {experiment }}-M_{\text {exact }} \\
\text { Equation } 1
\end{gathered}
$$

$M_{\text {error }}$ values are expressed in Da instead of ppm to ensure a linear evolution of $M_{\text {error }}$ errors along the $m / z$ axis (See Figure $2 A$ ).

\section{Selection of Hits}

Since the mass tolerance window for finding true hits T1 (e.g. set at $\pm 0.01 \mathrm{Da}$ for all the MSI data) can be large, a preselection of hits is necessary before fitting any model (Figure 1.4). The calculated mass errors of the hits (with Equation 1) are used for discarding false hits. As discussed previously, the assumption is that true hits display similar mass errors and the most populated errors should therefore contain a maximum of true hits. A kernel density estimation of the distribution of the errors is estimated by a Gaussian Kernel ${ }^{30}$ as illustrated in Figure 2.a. The hits of interest are finally selected within a certain range from the maximum of this distribution. This range will be called T2 (i.e. set at \pm 0.002 Da for all the MSI data treated here).

\section{Mass errors estimation and recalibration}

Hits selection reduces the probability to select false hits. Unfortunately, the application of simple linear regression function such as OLS (Ordinary Least Squares) leads to poor results since false hits are still present due to the complexity of biological signals. Therefore, RANSAC (Random Sample Consensus) algorithm ${ }^{31}$ is used for regression as it is more robust to outliers compared to OLS (Figure 2.b), the MS spectrum (MSI pixel) recalibration is performed by removing the mass errors estimated by the linear fit from each detected $m / z$ value. This step is made for each pixel of the MSI data. (Figure 1.5)
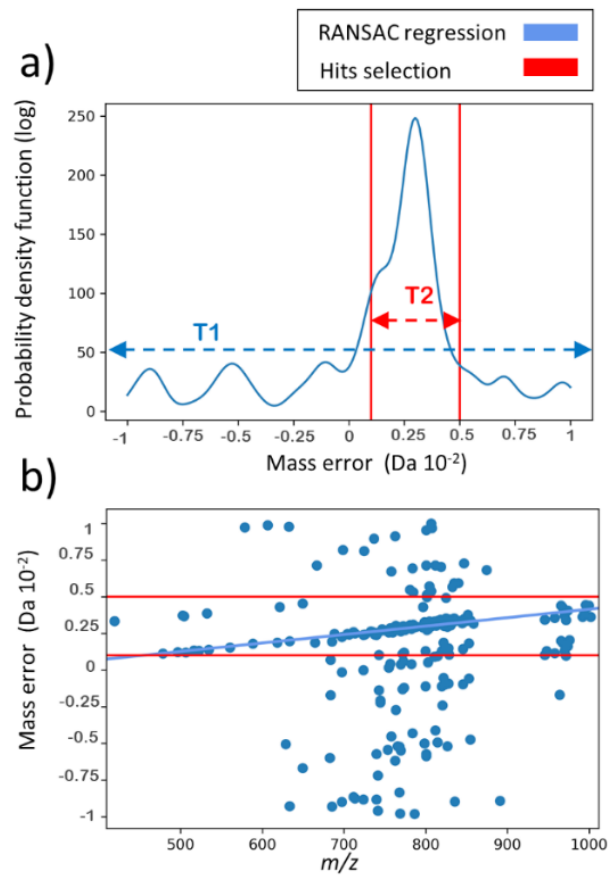

Figure 2. "Selection of hits" (a) and errors estimation (b) from the hits of a pixel from the MSI of the zebrafish slice.

The Figure 2 highlights the application of our algorithm to a single pixel of the zebrafish MSI data. The selection of hits based on the density estimation of the mass errors is shown in Figure 2.a, the selected hits are the points located within the red lines. The goal of this step is to filter out as many as possible of those hits which have too high error deviations from true hits, to maximize the chance of not including false hits during the model estimation. The RANSAC linear model is then estimated on the selected hits. Since many outliers are still present in the selected hits, the use of a robust linear estimator is necessary for detecting only true hits (Figure 2.b).

The reference implementation of the developed method is available at https://github.com/LaRoccaRaphael/MSI recalibrat ion. 
A more detailed version of the method is described in S2.

\section{Results and Discussion}

\section{Recalibration impact on data analysis}

To evaluate the efficiency of our internal calibration algorithm, the MSI data of a zebrafish slice imaged with a MALDI FT-ICR have been compared before and after recalibration. Observed mass shift is the result of the highly heterogeneous molecular composition of the organs and the tissues of this sample leading to different amounts of generated ions in the FT-ICR cell $^{15}$. Even for high accuracy instruments such as FT-ICR mass analyzer, the amplitude of mass shift is unknow for a given sample. For this reason, the values of $\mathrm{T} 1$ and $\mathrm{T} 2$ were set after investigating the mass shift of some pixels by using the plot as depicted in Figure 2.a, and then T1 and T2 values were adopted if an increase in annotations was observed by the quality plots (Figure 4). Finally, T1 was fixed at $\pm 0.01 \mathrm{Da}$ and T2 at $\pm 0.002 \mathrm{Da}$ for this dataset. However, too few public MSI datasets on zebrafish in positive mode were found on METASPACE. Therefore, we decide to generate the list of internal calibrating ions from Lipidmaps database ${ }^{32}$ with a selection of glycerophospholipids and sphingolipids ions ( $\mathrm{Na}^{+}$, $\mathrm{K}^{+}$and $\mathrm{H}^{+}$adducts). The Figure 3 shows a comparison of the average MS spectra of the image before and after recalibration for two different well-characterized ions (Figure $3 \mathrm{~A}$ and 3B), as well as the consequence on the reconstructed images of the ions for different tolerance (Figure $3 \mathrm{C}$ to $3 \mathrm{~J}$ ). The two peaks chosen for this evaluation are two phosphatidylcholine lipids, i.e. $\left[\mathrm{C}_{42} \mathrm{H}_{82} \mathrm{NO}_{8} \mathrm{P}+\mathrm{Na}\right]^{+}$and $\left[\mathrm{C}_{40} \mathrm{H}_{80} \mathrm{NO}_{8} \mathrm{P}+\mathrm{Na}\right]$ ${ }^{+}(\mathrm{m} / \mathrm{z}: 782.5670$ and 756.5514 , respectively).

Figure $3 \mathrm{~A}$ and $3 \mathrm{~B}$ clearly show a large distribution of the experimental $\mathrm{m} / \mathrm{z}$ values (in red) scattered over $7.5 \mathrm{mDa}$ and centered quite far from the exact theoretical $\mathrm{m} / \mathrm{z}(2.1 \%$ and $3.3 \%$ of the pixels are located within $1 \mathrm{ppm}$ from the exact mass of $\left[\mathrm{C}_{40} \mathrm{H}_{80} \mathrm{NO}_{8} \mathrm{P}+\mathrm{Na}\right]^{+}$and $\left[\mathrm{C}_{42} \mathrm{H}_{82} \mathrm{NO}_{8} \mathrm{P}+\mathrm{Na}\right]^{+}$, respectively). After recalibration (in green), the distribution of the $\mathrm{m} / \mathrm{z}$ is thinner (spread over $\pm 0.5 \mathrm{mDa}$ ) and more accurate $(97.35 \%$ and $98.74 \%$ of the pixels are located within $1 \mathrm{ppm}$ from the exact mass of $\left[\mathrm{C}_{40} \mathrm{H}_{80} \mathrm{NO}_{8} \mathrm{P}+\mathrm{Na}\right]^{+}$and $\left[\mathrm{C}_{42} \mathrm{H}_{82} \mathrm{NO}_{8} \mathrm{P}+\mathrm{Na}\right]^{+}$, respectively). The 2D intensity distribution (MSI image) of these two compounds extracted at $1 \mathrm{ppm}$ and $5 \mathrm{ppm}$ around their theoretical $\mathrm{m} / \mathrm{z}$ value are represented before calibration (Figure $3 \mathrm{C}$ and $3 \mathrm{G}$ for $1 \mathrm{ppm}$ and $3 \mathrm{D}$ and $3 \mathrm{H}$ for $5 \mathrm{ppm}$ ) and after recalibration (Figure $3 \mathrm{E}$ and $3 \mathrm{I}$ for $1 \mathrm{ppm}$ and $3 \mathrm{~F}$ and $3 \mathrm{G}$ for $5 \mathrm{ppm}$ ).
Before recalibration, a mass tolerance window of $5 \mathrm{ppm}$ was necessary to reconstruct the ion distribution as no image was obtained with 1 ppm. However, a large selection window increases the risk to get additional ions in the selected window, resulting in the creation of composite images. After recalibration, the vast majority of the signals of the investigated ions are included in the mass selection window of \pm $1 \mathrm{ppm}$ (green distributions). It results that these images are much more contrasted and more detailed, enhancing molecular description and interpretation. This highlights the impact of mass shift on the reconstruction of $\mathrm{m} / \mathrm{z}$ image. The comparison of MSI data before and after recalibration supports that our recalibration procedure avoids the loss in mass accuracy in the average MS signal due to mass shifts.
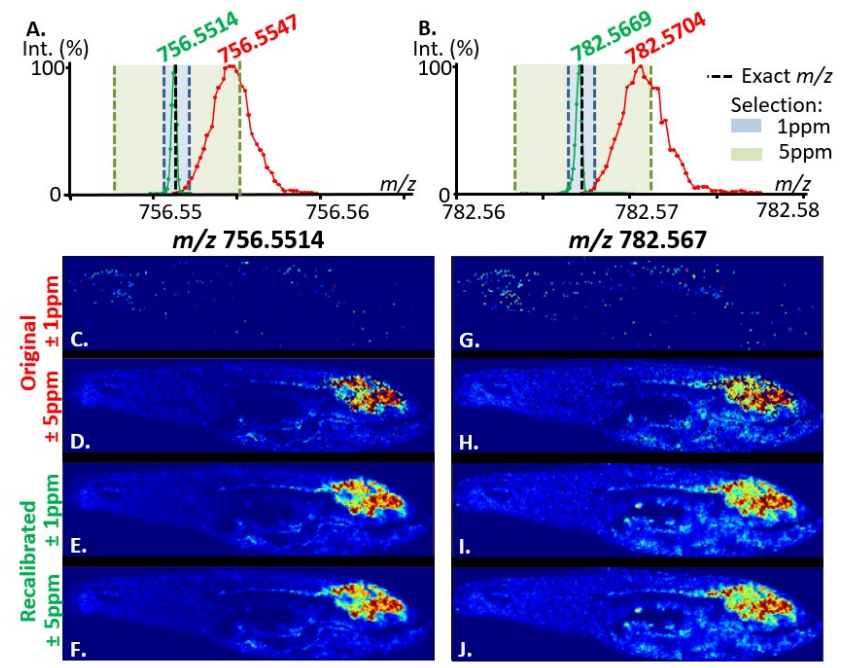

Figure 3. From a zebrafish MSI data. Comparison of the average spectra before and after recalibration for two different well-characterized ions $[\mathrm{C} 40 \mathrm{H} 80 \mathrm{NO} 8 \mathrm{P}+\mathrm{Na}]+$ and $[\mathrm{C} 42 \mathrm{H} 82 \mathrm{NO} 8 \mathrm{P}+\mathrm{Na}]+$, with a theoretical m/z of $756.5514 \mathrm{~m} / \mathrm{z}$ and $782.5670 \mathrm{~m} / \mathrm{z}$ respectively (Figure $3 \mathrm{~A}$ and $3 \mathrm{~B}$ ), as well as the consequence on the reconstructed images at \pm 1 $\mathrm{ppm}$ (Figure $3 \mathrm{C}$ to $3 \mathrm{G}$ and $3 \mathrm{E}$ to $3 \mathrm{I}$ ) and at $\pm 5 \mathrm{ppm}$ (Figure $3 \mathrm{D}$ to $3 \mathrm{H}$ and $3 \mathrm{~F}$ to $3 \mathrm{~J}$ ). The images are reconstructed from the theoretical mass of the two ions.

\section{Impact of Recalibration on the number of Molecular Annotations}

High mass accuracy is a crucial parameter for obtaining accurate annotations of molecular ions in $\mathrm{MSI}$ in absence of MS/MS (fragmentation information). As shown above, the recalibration increases the accuracy of the MS measurement (mass error from $4.3 \mathrm{ppm}$ to $0.12 \mathrm{ppm}$ ). Errors below 1 ppm are totally in line with the SolariX FTICR mass analyzer accuracy for single spectrum 
(considering adequate MS calibration). To quantify the impact of the recalibration on the annotation quality, both unprocessed (original) and recalibrated $\mathrm{MSI}$ data have been submitted to METASPACE for automatic annotation. Any METASPACE annotation is provided with its Metabolite-Signal Match score (MSM) that quantifies the likelihood of the measured signal to match the signal predicted for the molecule from a target database. The MSM score is computed by integrating (i) measure of spatial chaos of the ion image at the selected $m / z$, (ii) the similarity between the experimental and theoretical isotopic patterns and (iii) the spatial correlation between the reconstructed images of the isotopes. The estimation of false positives is made by employing a target-decoy approach where the decoy database contains implausible ions. From METASPACE, the target database can be selected among different popular options such as ChEBI (Chemical Entities of Biological Interest), HMDB (Human Metabolome Database), and LipidsMaps. The false discovery rate $^{19}$ (FDR) is estimated as the proportion of signals that matches the decoy database for that score against the signal that matches the target database for the same score. Mass shifts in MSI data should decrease the number of annotations for a given FDR by decreasing the true positive matches in the target database, by increasing the possibility of matching decoy signals and by decreasing the structure of the spatial localization of an ion (Figure 2). The number of METASPACE annotations for a given FDR appears then as an adequate criterion to evaluate the performance of a recalibration strategy. Original and recalibrated MSI are annotated by METASPACE, using the Lipidmaps database and considering the following adducts: $[\mathrm{M}+\mathrm{H}]^{+},[\mathrm{M}+\mathrm{Na}]^{+}$and $[\mathrm{M}+\mathrm{K}]^{+}$. Mass tolerances used for the identifications are 0.5, $1.0,1.5,2.0,2.5$ and from 3.0 to $10 \mathrm{ppm}$ with a step of $1 \mathrm{ppm}$. The performance of the recalibration is assessed by comparing the number of annotations of the original and recalibrated MSI (Figure 4). Classically, annotations with a FDR of $10 \%$ or lower are kept for analysis, as lower FDR corresponds to better annotation quality ${ }^{19}$. The most important increase would be at $1 \mathrm{ppm}$ with an FDR of $10 \%$ where more than 200 additional compounds were identified. This increase of annotation strongly supports the effectiveness of our recalibration strategy for reducing mass shift effect and for increasing the accuracy of MSI data.

\section{FDR and Tolerance Selection}

The evolution of annotation numbers with the tolerance for any FDR is due to two concomitant factors. First, the chances of matching decoy signals increase as the tolerance value increases, reducing the number of annotations. The second factor concerns the signal in the MSI data. When the tolerance value increases, the chance of matching isobaric or quasi-isobaric species instead of the expected signal also increases. If the isotopic pattern of these species is close to the suspected annotations, then the number of annotations will be falsely increased.

As a result, the variation in the number of annotations as a function of the mass tolerance depends on the predominance of these two factors, which is unpredictable for an unknown signal (Figure 4). However, the tolerance value in ppm should be low enough to optimize the number of annotations as it decreases the number of false positives. Therefore, the most interesting tolerance is the minimum tolerance value giving the highest number of annotations. FDR at $5 \%$ or $10 \%$ are commonly used for the interpretation of sample composition ${ }^{33}$. FDR below $10 \%$ are indicative of the quality of the sample signals, keeping only the most relevant identifications. However, higher FDR, such 20\% and $50 \%$, may be useful when considering annotations with low signal intensities. Indeed, as annotation scoring also depends on spatial structures and isotope distribution, low intensity signals have an impact on MSM scores and appear at higher FDR values. Since the recalibration shows a significant increase in annotations at higher FDR (Figure 4), the majority of the signal is probably weak in the initial data.

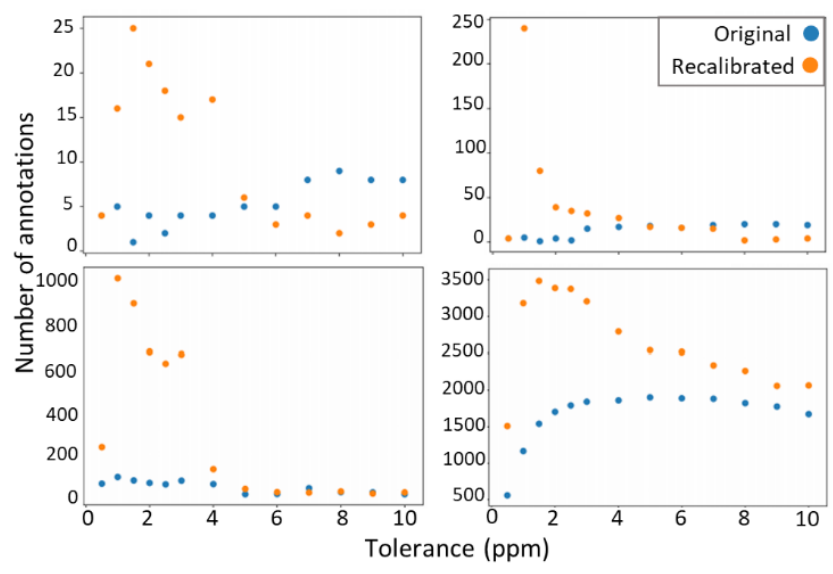

Figure 4. Number of METASPACE annotations of the original (blue dots) and recalibrated (orange dots) zebrafish MSI, for different mass tolerances (ppm). The number of annotations is shown for FDR $\leq 5 \%$, $\leq 10 \%$, $\leq 20 \%$ and $\leq 50 \%$.

\section{Evaluation on a large set of public MSI datasets}


The proposed algorithm was evaluated with 31 different MSI datasets, to assess its robustness under various experimental conditions. The recalibration of these datasets was performed using the settings detailed in the Material and Methods section. The recalibration parameters were determined using the same approach as explained in the section above "recalibration impact on data analysis" leading to the same values. Finally, the same parameters were used for T1 and T2, i.e. $\pm 0.01 \mathrm{Da}$ and $\pm 0.002 \mathrm{Da}$, respectively. Original and recalibrated MSI were then annotated by METASPACE. For MSI data in negative mode, only $[\mathrm{M}-\mathrm{H}]^{-}$and $[\mathrm{M}+\mathrm{Cl}]^{-}$ions were considered, whereas for MSI data in positive mode, $[\mathrm{M}+\mathrm{H}]^{+},[\mathrm{M}+\mathrm{Na}]^{+}$and $[\mathrm{M}+\mathrm{K}]^{+}$ions were taken into account. The mass tolerances set for the identification were $0.5 \mathrm{ppm}$ and every unit from 1.0 to $8.0 \mathrm{ppm}$. The performance of the recalibration was assessed by comparing the number of annotations of the original and recalibrated $\mathrm{MSI}$ as discussed above. All possible databases available on METASPACE platform were involved as target database to generate the $\mathrm{m} / \mathrm{z}_{\text {calib. }}$ list. Among them, the database that provided the best number of annotations on the unprocessed $\mathrm{MSI}$ data at a mass tolerance of 3 ppm and FDR of $10 \%$ was chosen (default settings in METASPACE). Therefore, it should be kept in mind that annotations may come from different databases depending on the dataset. Only the number of annotations at 5\% FDR and a tolerance of $1 \mathrm{ppm}$ were considered to normalize the evaluation of the algorithm's performance on this dataset. For some data, the recalibration method seemed to perform better for other values (see supporting info S3), but these were not taken into account because they were associated with a higher chance of false identifications (higher FDR or higher tolerance values). However, it could be useful to consider annotations at higher FDR values or higher tolerance for data mining as a perspective for this work. The benefit in terms of the number of annotations (i.e. the number of annotations in the recalibrated data minus the ones in the original data and thus the proportion of annotations gained) for each dataset is reported in Figure 5.
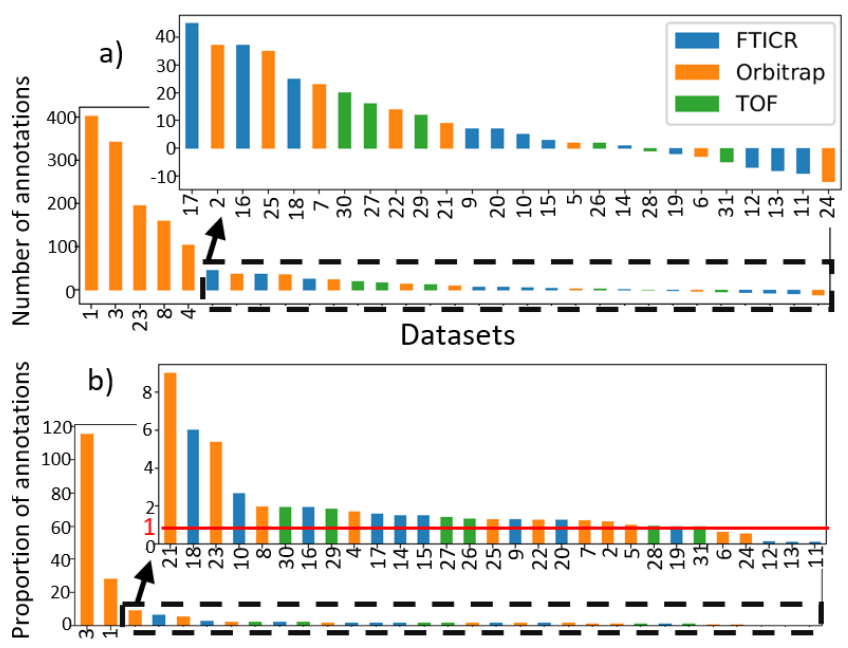

Figure 5. Bar plot representing a) the gain in the number of METASPACE annotations (FDR 5\%, 1 ppm tolerance) and b) proportion of annotations gained for each of 31 public MSI datasets after recalibrating with our method.

Our algorithm was able to increase the annotation numbers for $75 \%$ of the datasets, without any prior knowledge of the samples. For twelve of the recalibrated datasets, more than 20 additional annotations were obtained. This is particularly useful considering the fact that we consider high-quality annotations with FDR 5\% that often correspond to the most relevant molecular signals. Recalibration could not be performed on 3 datasets $(11,12$, and 13 in Figure 5 and S3), as the number of hits is under the applied threshold criterion of hits for processing recalibration (i.e. a minimum of 10 hits, see methods). After excluding these $3 \mathrm{MSI}$ datasets, the recalibration resulted in slightly fewer annotations for only $5 \mathrm{MSI}$ datasets, which can be explained either by poor quality of the datasets or by artefacts due to the recalibration.

Different hypotheses can be put forward to explain the cause of a poor recalibration. In a general context, the quality of recalibration in each pixel is related to the number of true hits taken into account for the estimation the recalibration function.

The MS signal quantity (i.e. amount of MS peaks over the noise) is the first key condition ensuring a robust recalibration of MSI data. Indeed, the recalibration of Orbitrap's MSI data, which globally contains more signals (more MS peaks) than other MSI data, gives the most impressive results (gaining from 20 up to 400 additional annotations), since more true hits are obtained. However, we cannot conclude that Orbitrap analyzers produce more MS signals than other mass analyzers in general, but this observation underlines the fact that recalibration performs 
better on data with more MS signals. As already mentioned in the method, the poor performance of the selection of true hits can also be attributed to the mass error - mass trend (e.g. amplitude of the slope of the linear regression and the nonlinearity evolution of the mass error in regard to the $m / z$ values) (Figure 2.b). However, taking these effects into account is challenging because the trend of the error may change from one pixel to another due to the heterogeneity of the signals in the MSI. Moreover, a sufficient number of true hits must be achieved to properly determine the error trend over the mass range. One solution to overcome these effects is to increase the mass tolerance for the hits selection T2 and T1 for the most extreme mass shift cases. However, it should be kept in mind that this could increase the number of false hits. Different mass tolerances were tested but did not give better results (data not shown). The poor recalibration is consequently mainly attributed to a lack of true matches between $\mathrm{m} / \mathrm{z}_{\text {exp. }}$ and $\mathrm{m} / \mathrm{z}_{\text {calib. }}$ due to weak signal. The values set for T1 and T2 seem to work well for all the MSI data analyzed in this work which can be explained by the fact that all data were acquired with mass analyzers providing high mass accuracy measurement. This is not surprising since METASPACE annotates MSI data at $\pm 3 p p m$ by default, promoting the submission of MSI acquired with high accuracy mass analyzers. Therefore, T1 and T2 could be used as constant for recalibrating MSI from METASPACE.

However, globally, it is essential to investigate certain recalibration conditions before applying the algorithm to other MSI data. These include (i) the assumption of the linear trend of the mass error as a function of $\mathrm{m} / \mathrm{z}$ (Figure 2.b), (ii) the amplitude of the slope of the error trend (Figure 2.b), (iii) the maximum deviation of the mass shift T1 and (iv) the mass selection tolerance for hits selection T2. These may depend on the initial acquisition and sample heterogeneity but also on the analyzer accuracy. Suggested quality plots (Figure 4, and Figures in S3) could be considered to validate the recalibration process on other MSI data and to help select the correct algorithm parameters in particular T1 and T2 (see methods and "Recalibration impact on data analysis"). Although the limitation of the number of hits seems to be the most crucial parameter to achieve good recalibration performance, this limitation will decrease in the future as the number of submitted datasets will increase. Indeed, enriching the database will cover more and more sample types and origins, thus increasing the quality of the $\mathrm{m} / \mathrm{z}_{\text {calib. lists }}$ generated. The sample signals will also likely be improved in the coming months and years, by increasing the ionization efficiency through the tuning of innovative ionization technologies such as laser-induced post-ionization (MALDI-2) ${ }^{34}$ or by improving the transmission between the ion source and the MS analyzer.

\section{Conclusion}

Pixel-to-pixel mass shift decreases the interpretability of MSI by affecting the image reconstruction of identified ions and by decreasing the quality and the number of annotations in HRMS. Those have thus a crucial impact on biological interpretation, reducing the capacity to locate and annotate potential biomarkers on biological samples.

Here, we report a new algorithm that recalibrate MSI data by adapting, at each pixel, the selection of endogenous signals for internal calibration. This method allows an optimal calibration of the different spatial regions of a MSI data decreasing the artefacts of mass shits during data analysis.

An evaluation of the method was proposed by using the number of annotations obtained on the METASPACE platform for the original and recalibrated $\mathrm{MSI}$ data as an indicator of recalibration performances. Through this evaluation, we tested the performance and robustness of our recalibration algorithm on 31 different $\mathrm{MSI}$ data from various origins. An increase in the number of annotations, indicating better calibration, was observed after recalibration for most of the investigated data (75\%). These results suggest that similar results could be obtained from different MSI acquired with similar MS analyzers.

However, this approach is not immune to certain limitations that could have an impact on recalibration performances. As discussed in this paper, these limitations depend either on the signals of the sample or on the algorithm parameters. In the first case, it can be expected that in a near future that the quality of the detected signal will increase thanks to the advances and progress made in ionization efficiency, MS instrumentation, and in METASPACE annotations coverage. In the second case, inspection of the algorithm parameters values should be considered for images with low recalibration performances. To this end, the proposed quality plots provide a useful visualization tool to investigate the effects of the optimal parameters on the recalibrated MSI data.

Finally, the integration of this data postprocessing in METASPACE is currently being considered since it uses many functionalities already available in METASPACE. In addition, it will allow the method to be tested on a large number of samples, which will highlight the best parameters to be used with this algorithm.

Ultra-high resolution instrument is not the only type mass spectrometer that suffers from mass 
shift, as a lower-resolution mass spectrometer may also be affected. In this case, an increase of T2 will be expected as it is directly impacted by the accuracy of the mass analyzer. The reported recalibration strategy can also be applied to each type of MS data, provided that each acquisition scan contains enough peaks to properly estimate the true mass error. It is also true for other MSbased approaches involving hyphenated separation methods, as the MS signal is expected to vary a lot during the acquisition (HPLC-MS, CEMS...). As for the MSI acquisition, the fluctuation of ions during the acquisition requires internal calibration to effectively reduce the mass shift effect.

In addition, more and more instruments are integrating ion-mobility feature into mass spectrometry. The MS recalibration strategy proposed in this work could be extended in a broader perspective to calibrate ion mobility values (CCS or mobility) through the use of mobility data from open-sources.

\section{ASSOCIATED CONTENT}

\section{Supporting Information}

The Supporting Information is availe sle free of charge on the ACS Publications website.

Description of the 31 METASPACE MSI data according to the initial uploader (S1), full detailed method section (S2),

plots for the 31 METASPACE MSI for the number of METASPACE annotations of the unprocessed (blue dots) and recalibrated (orange dots), for different mass tolerances (ppm). The number of annotations is shown for FDR $\leq 5 \%, \leq 10 \%, \leq 20 \%$ and $\leq 50 \%$ (S3). (PDF)

\section{AUTHOR INFORMATION}

\section{Corresponding Author}

* E-mail : raphael.larocca@uliege.be

\section{Notes}

The authors declare no competing financial interest.

\section{ACKNOWLEDGMENT}

We thank the METASPACE submitters who shared their data for this work: James McKenzie (ICL), Zoltan Takats (ICL), Dhaka Bhandari (JLU Giessen), Mario Kompauer (JLU Giessen), Shane Ellis (M4I), Dusan Velickovic (PNNL), Jessica Lukowski (PNNL), Lavigne Régis (U Rennes 1), Annapurna Pamreddy (UTHSA), Guanshi Zhang (UTHSA), Bindesh Shrestha (Waters), Emrys Jones (Waters).

The authors acknowledge financial support from the European Union's Horizon 2020 research and innovation program under grant agreement No. 731077 (EU FT-ICR MS project, INFRAIA-02-2017) and from the European Union and Wallonia program FEDER BIOMED HUB Technolohy Support (No. 2.2.1/996) for the funding of the SolariX XR 9.4T. The authors also thank the European Union's Horizon 2020 program (EURLipids Interreg Eurogio MeuseRhine project supported by the European Regional Development Fund (FEDER)).

\section{REFERENCES}

(1) Vaysse, P.-M.; Heeren, R. M. A.; Porta, T.; Balluff, B. Mass Spectrometry Imaging for Clinical Research Latest Developments, Applications, and Current Limitations. Analyst 2017, 142 (15), 2690-2712. https://doi.org/10.1039/C7AN00565B.

(2) Caprioli, R. M.; Farmer, T. B.; Gile, J. Molecular Imaging of Biological Samples: Localization of Peptides and Proteins Using MALDI-TOF MS. Anal. Chem. 1997, 69 (23), 4751-4760. https://doi.org/10.1021/ac970888i.

(3) Gode, D.; Volmer, D. A. Lipid Imaging by Mass Spectrometry - a Review. Analyst 2013, 138 (5), 1289. https://doi.org/10.1039/c2an36337b.

(4) Khatib-Shahidi, S.; Andersson, M.; Herman, J. L.; Gillespie, T. A.; Caprioli, R. M. Direct Molecular Analysis of Whole-Body Animal Tissue Sections by Imaging MALDI Mass Spectrometry. Anal. Chem. 2006, 78 (18), 6448-6456. https://doi.org/10.1021/ac060788p.

(5) Stoeckli, M.; Staab, D.; Schweitzer, A. Compound and Metabolite Distribution Measured by MALDI Mass Spectrometric Imaging in Whole-Body Tissue Sections. International Journal of Mass Spectrometry 2007, $260 \quad(2-3), \quad 195-202$. https://doi.org/10.1016/j.ijms.2006.10.007.

(6) Yang, J. Y.; Phelan, V. V.; Simkovsky, R.; Watrous, J. D.; Trial, R. M.; Fleming, T. C.; Wenter, R.; Moore, B. S.; Golden, S. S.; Pogliano, K.; Dorrestein, P. C. Primer on Agar-Based Microbial Imaging Mass Spectrometry. Journal of Bacteriology 2012, 194 (22), 6023-6028. https://doi.org/10.1128/JB.00823-12.

(7) Zaima, N.; Goto-Inoue, N.; Hayasaka, T.; Setou, M. Application of Imaging Mass Spectrometry for the Analysis of Oryza Sativa Rice. Rapid Commun. Mass Spectrom. 2010, 24 (18), 2723-2729. https://doi.org/10.1002/rcm.4693.

(8) Neumann, E. K.; Ellis, J. F.; Triplett, A. E.; Rubakhin, S. S.; Sweedler, J. V. Lipid Analysis of 30000 Individual Rodent Cerebellar Cells Using High-Resolution Mass Spectrometry. Anal. Chem. 2019, 91 (12), 78717878. https://doi.org/10.1021/acs.analchem.9b01689.

(9) Boggio, K. J.; Obasuyi, E.; Sugino, K.; Nelson, S. B.; Agar, N. Y.; Agar, J. N. Recent Advances in Single-Cell MALDI Mass Spectrometry Imaging and Potential Clinical Impact. Expert Review of Proteomics 2011, 8 (5), 591-604. https://doi.org/10.1586/epr.11.53.

(10) Cooks, R. G. Ambient Mass Spectrometry. Science 2006, 311 (5767), 1566-1570. https://doi.org/10.1126/science.1119426.

(11) Brunelle, A.; Touboul, D.; Laprévote, O. Biological Tissue Imaging with Time-of-Flight Secondary Ion Mass Spectrometry and Cluster Ion Sources. J. Mass Spectrom. 2005, 40 (8), 985-999. https://doi.org/10.1002/jms.902.

(12) Alexandrov, T. MALDI Imaging Mass Spectrometry: Statistical Data Analysis and Current Computational Challenges. BMC Bioinformatics 2012, 13 (S16), S11. https://doi.org/10.1186/1471-2105-13S16-S11. 
(13) Buchberger, A. R.; DeLaney, K.; Johnson, J.; Li, L. Mass Spectrometry Imaging: A Review of Emerging Advancements and Future Insights. Anal. Chem. 2018 , 90 (1), 240-265 https://doi.org/10.1021/acs.analchem.7b04733.

(14) Schwudke, D.; Schuhmann, K.; Herzog, R.; Bornstein, S. R.; Shevchenko, A. Shotgun Lipidomics on High Resolution Mass Spectrometers. Cold Spring Harbor Perspectives in Biology 2011, 3 (9), a004614a004614. https://doi.org/10.1101/cshperspect.a004614.

(15) Nikolaev, E. N.; Kostyukevich, Y. I.; Vladimirov, G. N. Fourier Transform Ion Cyclotron Resonance (FT ICR) Mass Spectrometry: Theory and Simulations: FT ICR MS. Mass Spec Rev 2016, 35 (2), 219-258. https://doi.org/10.1002/mas.21422.

(16) Smith, D. F.; Kharchenko, A.; Konijnenburg, M.; Klinkert, I.; Paša-Tolić, L.; Heeren, R. M. A. Advanced Mass Calibration and Visualization for FT-ICR Mass Spectrometry Imaging. J. Am. Soc. Mass Spectrom. 2012, 23 (11), 1865-1872. https://doi.org/10.1007/s13361-012-0464-1.

(17) Kozhinov, A. N.; Zhurov, K. O.; Tsybin, Y. O. Iterative Method for Mass Spectra Recalibration via Empirical Estimation of the Mass Calibration Function for Fourier Transform Mass Spectrometry-Based Petroleomics. Anal. Chem. 2013, 85 (13), 6437-6445. https://doi.org/10.1021/ac400972y.

(18) Burton, R. D.; Matuszak, K. P.; Watson, C. H.; Eyler, J. R. Exact Mass Measurements Using a 7 Tesla Fourier Transform Ion Cyclotron Resonance Mass Spectrometer in a Good Laboratory Practices-Regulated Environment. J Am Soc Mass Spectrom 1999, 10 (12), 1291-1297. 0305(99)00106-3.

(19) Palmer, A.; Phapale, P.; Chernyavsky, I.; Lavigne, R.; Fay, D.; Tarasov, A.; Kovalev, V.; Fuchser, J.; Nikolenko, S.; Pineau, C.; Becker, M.; Alexandrov, T. FDR-Controlled Metabolite Annotation for HighResolution Imaging Mass Spectrometry. Nat Methods 2017, 14 (1), 57-60. https://doi.org/10.1038/nmeth.4072.

(20) Alexandrov, T. Spatial Metabolomics and Imaging Mass Spectrometry in the Age of Artificial Intelligence. Annu. Rev. Biomed. Data Sci. 2020, 3 (1), 61-87. https://doi.org/10.1146/annurev-biodatasci011420-031537.

(21) Gross, J. H. Mass Spectrometry; Springer Berlin Heidelberg: Berlin, Heidelberg, 2011. https://doi.org/10.1007/978-3-642-10711-5.

(22) Tiquet, M.; La Rocca, R.; van Kruining, D.; Martinez-Martinez, P.; Eppe, G.; De Pauw, E.; Quinton, L.; Far, J. Mass Spectrometry Imaging Using Dynamically Harmonized FT-ICR at Million Resolving Power: Rationalizing and Optimizing Sample Preparation and Instrumental Parameters; preprint; 2020. https://doi.org/10.26434/chemrxiv.13013900.v1.

(23) Kooijman, P. C.; Nagornov, K. O.; Kozhinov, A. N.; Kilgour, D. P. A.; Tsybin, Y. O.; Heeren, R. M. A.; Ellis, S. R. Increased Throughput and Ultra-High Mass Resolution in DESI FT-ICR MS Imaging through NewGeneration External Data Acquisition System and Advanced Data Processing Approaches. Sci Rep 2019 , 9 (1), 8. https://doi.org/10.1038/s41598-018-36957-1.

(24) Boskamp, T.; Lachmund, D.; Casadonte, R.; Hauberg-Lotte, L.; Kobarg, J. H.; Kriegsmann, J.; Maass, P. Using the Chemical Noise Background in MALDI Mass Spectrometry Imaging for Mass Alignment and
Calibration. Anal. Chem. 2020, 92 (1), 1301-1308. https://doi.org/10.1021/acs.analchem.9b04473.

(25) Tracy, M. B.; Chen, H.; Weaver, D. M.; Malyarenko, D. I.; Sasinowski, M.; Cazares, L. H.; Drake, R. R.; Semmes, O. J.; Tracy, E. R.; Cooke, W. E. Precision Enhancement of MALDI-TOF MS Using

High Resolution Peak Detection and Label-Free Alignment. Proteomics 2008, 8 (8), 1530-1538. https://doi.org/10.1002/pmic.200701146.

(26) Brochu, F.; Plante, P.-L.; Drouin, A.; Gagnon, D.; Richard, D.; Durocher, F.; Diorio, C.; Marchand, M.; Corbeil, J.; Laviolette, F. Mass Spectra Alignment Using Virtual Lock-Masses. Sci Rep 2019, 9 (1), 8469. https://doi.org/10.1038/s41598-019-44923-8.

(27) Ràfols, P.; Castillo, E. del; Yanes, O.; Brezmes, J.; Correig, X. Novel Automated Workflow for Spectral Alignment and Mass Calibration in MS Imaging Using a Sputtered Ag Nanolayer. Analytica Chimica Acta 2018, 1022, 61-69. https://doi.org/10.1016/j.aca.2018.03.031.

(28) Kulkarni, P.; Kaftan, F.; Kynast, P.; Svatoš, A.; Böcker, S. Correcting Mass Shifts: A Lock Mass-Free Recalibration Procedure for Mass Spectrometry Imaging Data. Anal Bioanal Chem 2015, 407 (25), 7603-7613. https://doi.org/10.1007/s00216-015-8935-4.

(29) Schramm, T.; Hester, Z.; Klinkert, I.; Both, J.-P.; Heeren, R. M. A.; Brunelle, A.; Laprévote, O.; Desbenoit, N.; Robbe, M.-F.; Stoeckli, M.; Spengler, B.; Römpp, A. ImzML - A Common Data Format for the Flexible Exchange and Processing of Mass Spectrometry Imaging Data. Journal of Proteomics 2012, 75 (16) 5106-5110. https://doi.org/10.1016/j.jprot.2012.07.026.

(30) Ledl, T. Kernel Density Estimation: Theory and Application in Discriminant Analysis. AJS 2016, 33 (3), 267-279. https://doi.org/10.17713/ajs.v33i3.441.

(31) Fischler, M. A.; Bolles, R. C. Random Sample Consensus: A Paradigm for Model Fitting with Applications to Image Analysis and Automated Cartography. Commun. ACM 1981, 24 (6), 381-395. https://doi.org/10.1145/358669.358692.

(32) Sud, M.; Fahy, E.; Cotter, D.; Brown, A.; Dennis, E. A.; Glass, C. K.; Merrill, A. H.; Murphy, R. C.; Raetz, C. R. H.; Russell, D. W.; Subramaniam, S. LMSD: LIPID MAPS Structure Database. Nucleic Acids Research 2007, 35 (Database), D527-D532. https://doi.org/10.1093/nar/gkl838.

(33) Alexandrov, T.; Ovchinnikova, K.; Palmer, A.; Kovalev, V.; Tarasov, A.; Stuart, L.; Nigmetzianov, R.; Fay, D.; Key METASPACE contributors; Gaudin, M.; Lopez, C. G.; Vetter, M.; Swales, J.; Bokhart, M.; Kompauer, M.; McKenzie, J.; Rappez, L.; Velickovic, D.; Lavigne, R.; Zhang, G.; Thinagaran, D.; Ruhland, E.; Sans, M.; Triana, S.; Sammour, D. A.; Aboulmagd, S.; Bagger, C.; Strittmatter, N.; Rigopoulos, A.; Gemperline, E.; Joensen, A. M.; Geier, B.; Quiason, C.; Weaver, E.; Prasad, M.; Balluff, B.; Nagornov, K.; Li, L.; Linscheid, M.; Hopf, C.; Heintz, D.; Liebeke, M.; Spengler, B.; Boughton, B.; Janfelt, C.; Sharma, K.; Pineau, C.; Anderton, C.; Ellis, S.; Becker, M.; Pánczél, J.; Violante, G. D.; Muddiman, D.; Goodwin, R.; Eberlin, L.; Takats, Z.; Shahidi-Latham, S. METASPACE: A CommunityPopulated Knowledge Base of Spatial Metabolomes in Health and Disease; preprint; Systems Biology, 2019. https://doi.org/10.1101/539478.

(34) Soltwisch, J.; Kettling, H.; Vens-Cappell, S.; Wiegelmann, M.; Muthing, J.; Dreisewerd, K. Mass Spectrometry Imaging with Laser-Induced 
Postionization. Science 2015, 348 (6231), 211-215.

https://doi.org/10.1126/science.aaa1051. 